\title{
CHSH-Type Inequality Involving Commuting Continuous Variables
}

\author{
Andrea Valdés-Hernández *, Ana María Cetto iD and Luis de la Peña \\ Instituto de Física, Universidad Nacional Autónoma de México, Mexico City A. P. 20-364, Mexico; \\ ana@fisica.unam.mx (A.M.C.); luis@fisica.unam.mx (L.d.1.P.) \\ * Correspondence: andreavh@fisica.unam.mx
}

Received: 14 February 2019; Accepted: 23 April 2019; Published: 25 April 2019

\begin{abstract}
The correlation of projections of the momentum operators of two particles is used to derive a quantum inequality for continuous variables, which must be satisfied by any bipartite system in a pure state. This inequality resembles a Clauser-Horne-Shimony-Holt (CHSH)-type inequality except for additional terms related to the imaginary component of the weak value of the momentum, which normally remains concealed in the usual quantum description but turns out to be of relevance for entangled states. Our results shed new light on the link between noncommutativity, entanglement and nonlocality of the quantum description.
\end{abstract}

Keywords: bipartite entanglement; weak values of linear momentum; flow and diffusive velocities; CHSH-type inequalities

\section{Introduction}

A distinctive feature of pure entangled states of a bipartite system is that they violate the Clauser-Horne-Shimony-Holt (CHSH) inequality, derived for discrete, non-commuting operators [1,2]. Entanglement and violation of the $\mathrm{CHSH}$ inequality are therefore considered synonymous [3]. This well-known fact is used here as a guide to take further the intimate connection between entanglement and the (imaginary part of) the weak value of the linear momentum [4], by means of an inequality involving a $\mathrm{CHSH}$-type operator for continuous, commuting momentum variables, which serves to certify entanglement.

As a background for the present work, we note that the real and imaginary parts of the linear momentum weak values [5,6] - which correspond to the flow and diffusive (or osmotic) velocity components (see e.g., $[7,8]$ ) - have been the subject of various efforts aimed at exploring quantum features in novel ways [9-19]. In particular, the diffusive velocity has been shown to play a prominent role in different forms of entanglement indicators for pure bipartite states [4].

In light of these results, here we construct an inequality that must be satisfied by any bipartite system in a pure quantum state. This inequality, derived for continuous variables associated with projections of the momentum operators of the two particles, has exactly the structure of the (standard) $\mathrm{CHSH}$ inequality except for the presence of extra correlations that vanish whenever the state is separable. Such correlation terms depend exclusively on the diffusive-velocity component associated with each one of the particles. Hence the diffusive velocity is ultimately responsible for the violation of the CHSH-type inequality, thus serving to certify the non-factorizability of the probability distribution, which is a signature of entanglement. Our results represent thus a step forward in the understanding of the link between entanglement and nonlocality of the quantum description.

The structure of the paper is as follows. In Section 2 we introduce the flow and diffusive velocities as the real and imaginary parts, respectively, of the momentum weak values. We further give arguments in support of identifying the diffusive velocity component as a hallmark of quantumness. 
In Section 3 we briefly review previously reported entanglement criteria based on the diffusive velocity, which are of relevance for the present work. We further proceed to construct a CHSH-type operator for continuous variables and derive an inequality for the mean value of this operator, which must be satisfied by construction. By neglecting the diffusive-velocity terms we arrive at a CHSH-type inequality. The paper concludes with a brief discussion of the results and possible perspectives, in Section 4.

\section{Flow and Diffusive Velocities}

Let us consider a two-particle pure state $|\psi(t)\rangle$ described by the wave function

$$
\left\langle x_{1} x_{2} \mid \psi\right\rangle=\psi\left(x_{1}, x_{2}, t\right)=\sqrt{\rho\left(x_{1}, x_{2}, t\right)} e^{i S\left(x_{1}, x_{2}, t\right)}
$$

where $\rho=|\psi|^{2}$ and $S$ is a real function. We assume that the system is bounded so that the wave function vanishes at infinity. Let $\hat{\boldsymbol{p}}_{i}$ be the linear momentum operator of the $i$-th particle $(i=1,2)$ with mass $m_{i}$; direct calculation gives

$$
\hat{\boldsymbol{p}}_{i} \psi=-i \hbar \nabla_{i} \psi=m_{i}\left(\boldsymbol{v}_{i}-i \boldsymbol{u}_{i}\right) \psi
$$

with the (real) velocity vectors $\boldsymbol{v}_{i}$ and $\boldsymbol{u}_{i}$ given respectively by

$$
\boldsymbol{v}_{i}\left(x_{1}, x_{2}, t\right)=\frac{\hbar}{m_{i}} \nabla_{i} S, \quad \boldsymbol{u}_{i}\left(x_{1}, x_{2}, t\right)=\frac{\hbar}{2 m_{i}} \frac{\nabla_{i} \rho}{\rho} .
$$

In what follows we refer as usual to $v$ as the flow velocity, and to $u$ as the diffusive velocity.

In terms of the weak value [5,6] of the momentum operator (with preselection state $|\psi(t)\rangle$ and postselection state $\left|x_{1} x_{2}\right\rangle$ ),

$$
\left\langle\hat{\boldsymbol{p}}_{i}\right\rangle_{\mathrm{w}} \equiv \frac{\left\langle\boldsymbol{x}_{1} x_{2}\left|\hat{\boldsymbol{p}}_{i}\right| \psi\right\rangle}{\left\langle\boldsymbol{x}_{1} \boldsymbol{x}_{2} \mid \psi\right\rangle}=-\frac{i \hbar \nabla_{i} \psi}{\psi},
$$

the individual flow and diffusive velocities take the form

$$
\boldsymbol{v}_{i}=\frac{1}{m_{i}} \operatorname{Re}\left\langle\hat{\boldsymbol{p}}_{i}\right\rangle_{\mathrm{w}}, \quad \boldsymbol{u}_{i}=-\frac{1}{m_{i}} \operatorname{Im}\left\langle\hat{\boldsymbol{p}}_{i}\right\rangle_{\mathrm{w}} .
$$

The fact that $\boldsymbol{v}_{i}$ and $\boldsymbol{u}_{i}$ correspond to the real and imaginary parts of the weak value of a Hermitian operator allows us to disclose some of their respective roles in expressions for quantum expectation values [4]. In general, for a Hermitian operator $\hat{A}$ it can be shown that

$$
\left\langle\operatorname{Re}\langle\hat{A}\rangle_{\mathrm{w}}\right\rangle=\langle\hat{A}\rangle_{\mathrm{q}}, \quad\left\langle\operatorname{Im}\langle\hat{A}\rangle_{\mathrm{w}}\right\rangle=0,
$$

where $\langle\hat{A}\rangle_{\mathrm{w}}=\frac{\left\langle x_{1} x_{2}|\hat{A}| \psi\right\rangle}{\left\langle x_{1} x_{2} \mid \psi\right\rangle},\langle\hat{A}\rangle_{\mathrm{q}}$ denotes the quantum expectation value

$$
\langle\hat{A}\rangle_{\mathrm{q}}=\langle\psi|\hat{A}| \psi\rangle=\int \psi^{*} \hat{A} \psi d x_{1} d x_{2}
$$

and $\langle\cdot\rangle$ stands for the statistical mean value defined as

$$
\langle\cdot\rangle=\int(\cdot) \rho d x_{1} d x_{2}
$$

It seems convenient to make here some remarks regarding the meaning of the mean values just introduced. The first one, namely $\langle\cdot\rangle_{\mathrm{W}}{ }^{\prime}$, stands for the weak value $\langle\cdot\rangle_{\mathrm{W}}=\frac{\left\langle x_{1} x_{2}|\cdot| \psi\right\rangle}{\left\langle x_{1} x_{2} \mid \psi\right\rangle}$, and the mean-value notation originates in the fact that the weak values represent averaged values of weak measurements, over pre and post-selected (sub)ensembles $[10,20]$. In contrast, the (usual) quantum expectation values, 
$\langle\hat{A}\rangle_{\mathrm{q}}$, are obtained by averaging over an ensemble of systems prepared in state $|\psi\rangle$ and involve projective (von Neumann) measurements. Finally, the statistical mean value, $\langle\cdot\rangle$, differs from the quantum expectation value in that it refers to a mean value of c-numbers (instead of q-numbers), and averages over an ensemble of systems described by the probability distribution density $\rho=\psi^{*} \psi$.

From Equations (5) and (6) it follows that $v_{i}$ plays the role of a local (defined at each point $\left(x_{1}, x_{2}\right)$ ) velocity, such that its average over the entire space results in

$$
\left\langle\hat{\boldsymbol{p}}_{i}\right\rangle_{\mathrm{q}}=m_{i}\left\langle\boldsymbol{v}_{i}\right\rangle .
$$

In line with refs. $[8,16]$, the flow velocity represents a mean velocity averaged over an ensemble of individual particles, whereas in Bohmian mechanics [10,21-23] it is interpreted as the actual velocity of the $i$-th particle describing the trajectory $x_{i}(t)$. Further, in the quantum formalism it represents the velocity associated with the probability current $j_{i}=\rho v_{i}$ appearing in the continuity equation $\frac{\partial \rho}{\partial t}+\sum_{i} \nabla_{i} \cdot j_{i}=0$.

As for $\boldsymbol{u}_{i}$, Equations (5) and (6) imply that

$$
\left\langle\boldsymbol{u}_{i}\right\rangle=0,
$$

so that it does not contribute to the expectation value of the momentum operator. Physically, this is consistent with the fact that, unlike $\boldsymbol{v}_{i}, \boldsymbol{u}_{i}$ is not related to a translational motion but rather to the diffusive or dispersive behavior of the ensemble, as will be discussed below. The diffusive velocity surfaces instead in bilinear expressions such as $\left\langle\hat{p}_{i} \cdot \hat{p}_{j}\right\rangle_{\mathrm{q}}$, which reads

$$
\left\langle\hat{\boldsymbol{p}}_{i} \cdot \hat{\boldsymbol{p}}_{j}\right\rangle_{\mathrm{q}}=m_{i} m_{j}\left(\left\langle\boldsymbol{v}_{i} \cdot \boldsymbol{v}_{j}\right\rangle+\left\langle\boldsymbol{u}_{i} \cdot \boldsymbol{u}_{j}\right\rangle\right) .
$$

This is just one instance of the general observation that the imaginary part of the weak value of a Hermitian operator may become relevant in the calculation of quantum correlations between Hermitian operators corresponding to dynamical variables of the individual subsystems [4].

It is convenient at this point to recall a few facts about the diffusive velocity, considering its importance for the present discussion. The diffusive (or osmotic) velocity has been largely studied in several contexts, notably in stochastic electrodynamics (see [8] and references therein) and stochastic quantum mechanics $[7,24-26]$, in the old hydrodynamic model of quantum mechanics $[27,28]$ (albeit collaterally), and more recently in the weak-value context $[15,18,19]$. A first hint of its importance in the general quantum context is that the mean value of the basic commutator becomes expressed naturally in terms of this velocity [4],

$$
\left\langle\left[\hat{x}_{i}, \hat{p}_{i}\right]\right\rangle_{\mathrm{q}}=\left\langle\hat{x}_{i} \cdot \hat{\boldsymbol{p}}_{i}-\hat{\boldsymbol{p}}_{i} \cdot \hat{\boldsymbol{x}}_{i}\right\rangle_{\mathrm{q}}=-2 i m_{i}\left\langle\boldsymbol{x}_{i} \cdot \boldsymbol{u}_{i}\right\rangle,
$$

whence it represents a direct measure of the quantum fluctuations. This can be seen more clearly by putting $i=j$ in Equation (11), which gives for the dispersion of $\hat{p}_{i}$ :

$$
\sigma_{\hat{\boldsymbol{p}}_{i}}^{2}=\left\langle\hat{\boldsymbol{p}}_{i}^{2}\right\rangle_{\mathrm{q}}-\left\langle\hat{\boldsymbol{p}}_{i}\right\rangle_{\mathrm{q}}^{2}=m_{i}^{2}\left(\sigma_{\boldsymbol{v}_{i}}^{2}+\left\langle\boldsymbol{u}_{i}^{2}\right\rangle\right) \geq m_{i}^{2}\left\langle\boldsymbol{u}_{i}^{2}\right\rangle>0,
$$

where the last (strict) inequality holds for any bounded system. This confirms that the diffusive velocity is intimately related to the presence of quantum (irreducible) fluctuations. ${ }^{1}$

1 Even if the expressions just discussed do not throw light on the origin of the quantum fluctuations-the important point here being that they exist-some words about this point seem advisable. The common stand is that the quantum fluctuations refer to an irreducible (noncausal) phenomenon, which makes it apparently unnecessary to go beyond the recognition of their existence. However, in different works a cause is proposed for them, which may differ from author to author. We recall in particular the proposal discussed in ref. [8] and references therein, where it is shown that the origin of the 
Further, $u$ appears in another characteristically quantum function, namely the ubiquitous quantum potential $V_{Q}$, prominent in Bohm's theory and in stochastic quantum mechanics, and given (in the $N$-particle scenario) by $[4,29]$

$$
V_{Q} \equiv-\hbar^{2} \sum_{k=1}^{N} \frac{1}{2 m_{k}} \frac{\nabla_{k}^{2} \sqrt{\rho}}{\sqrt{\rho}}=-\frac{1}{2} \sum_{k=1}^{N}\left(m_{k} \boldsymbol{u}_{k}^{2}+\hbar \nabla \cdot \boldsymbol{u}_{k}\right)
$$

Remarkably, $u$ has also been shown to be intimately connected with other distinctive quantum features, in particular with entanglement in the bipartite case [4,8]. In short, one can say that the diffusive velocity, proper to all quantum systems (save the free particle with fixed energy), is a hallmark of quantumness.

\section{An Inequality Involving a CHSH-Type Operator for Continuous Variables}

The connection between the diffusive velocities and entanglement criteria has been recently explored in [4]. We briefly present the results reported in [4] as a background for the present discussion, and then proceed to obtain our main result.

The bipartite state (1) is said to be non-entangled whenever it can be described by a factorizable wave function,

$$
\psi\left(x_{1}, x_{2}, t\right)=\psi_{1}\left(x_{1}, t\right) \psi_{2}\left(x_{2}, t\right),
$$

with $\psi_{i}=\sqrt{\rho_{i}} \exp \left(i S_{i}\right)$, so that the following two conditions are simultaneously met:

1. $\rho\left(x_{1}, x_{2}, t\right)=\rho_{1}\left(x_{1}, t\right) \rho_{2}\left(x_{2}, t\right)$,

2. $S\left(x_{1}, x_{2}, t\right)=S_{1}\left(x_{1}, t\right)+S_{2}\left(x_{2}, t\right)$.

If condition 1 is met we say that the state is 'A-separable'. Otherwise it is said to be 'A-entangled', indicating that the entanglement is encoded in the non-factorizability of the amplitude of $\rho$. Analogously, if condition 2 is met we say that the state is 'P-separable'; otherwise it is said to be 'P-entangled', stressing that the entanglement is encoded in the non-additivity of the phase $S$.

In [4] it was shown that

$$
\begin{array}{r}
\left\langle\boldsymbol{u}_{1} \cdot \boldsymbol{u}_{2}\right\rangle \neq 0 \Rightarrow \psi \text { is A-entangled }, \\
\left\langle\boldsymbol{u}_{1} \cdot \boldsymbol{v}_{2}\right\rangle=\left\langle\boldsymbol{u}_{2} \cdot \boldsymbol{v}_{1}\right\rangle \neq 0 \Rightarrow \psi \text { is P-entangled. }
\end{array}
$$

Thus, when the absolute value is taken in Equation (11) so that

$$
\left|\left\langle\hat{\boldsymbol{p}}_{1} \cdot \hat{\boldsymbol{p}}_{2}\right\rangle_{\mathrm{q}}\right| \leq m_{1} m_{2}\left(\left|\left\langle\boldsymbol{v}_{1} \cdot \boldsymbol{v}_{2}\right\rangle\right|+\left|\left\langle\boldsymbol{u}_{1} \cdot \boldsymbol{u}_{2}\right\rangle\right|\right),
$$

we see that the correlation between the diffusive velocities increases the upper bound of the quantum momentum correlations when there is A-entanglement. ${ }^{2}$ This observation evokes the $\mathrm{CHSH}$ inequality, in the sense that its usefulness in certifying entanglement relies on the fact that quantum correlations exceed the classical upper bound of the expectation value of an appropriate operator [30]. This suggests we should take the parallelism further and investigate whether a similar inequality involving a CHSH-type operator for continuous (momentum) variables can be established. As will become clear below, such inequality would serve to certify the presence of (A-)entanglement, and to identify where and how exactly the nonclassical information of the system is encoded.

quantum fluctuations in atomic systems can be ascribed to their permanent interaction with the zero-point radiation field; this constitutes the causal, realistic and objective theory known as stochastic electrodynamics.

2 Notice that correlations of the form $\left\langle\boldsymbol{u}_{i} \cdot \boldsymbol{v}_{j}\right\rangle$, witnessing P-entanglement, do not contribute explicitly to $\left\langle\hat{\boldsymbol{p}}_{1} \cdot \hat{\boldsymbol{p}}_{2}\right\rangle_{\mathrm{q}}$. 
In order to construct the desired inequality, we first consider the CHSH-type operator

$$
\hat{\mathrm{C}}=\left(\boldsymbol{a} \cdot \hat{\boldsymbol{p}}_{1}\right)\left(\boldsymbol{b} \cdot \hat{\boldsymbol{p}}_{2}\right)+\left(\boldsymbol{a} \cdot \hat{\boldsymbol{p}}_{1}\right)\left(\boldsymbol{b}^{\prime} \cdot \hat{\boldsymbol{p}}_{2}\right)+\left(\boldsymbol{a}^{\prime} \cdot \hat{\boldsymbol{p}}_{1}\right)\left(\boldsymbol{b} \cdot \hat{\boldsymbol{p}}_{2}\right)-\left(\boldsymbol{a}^{\prime} \cdot \hat{\boldsymbol{p}}_{1}\right)\left(\boldsymbol{b}^{\prime} \cdot \hat{\boldsymbol{p}}_{2}\right),
$$

with $\boldsymbol{a}, \boldsymbol{a}^{\prime}, \boldsymbol{b}, \boldsymbol{b}^{\prime}$ arbitrary unit vectors. Now, resorting to Equations (2), (6) and (7) with $\hat{A}=$ $\left(\boldsymbol{a} \cdot \hat{\boldsymbol{p}}_{1}\right)\left(\boldsymbol{b} \cdot \hat{\boldsymbol{p}}_{2}\right)$, we get

$$
\left\langle\left(\boldsymbol{a} \cdot \hat{\boldsymbol{p}}_{1}\right)\left(\boldsymbol{b} \cdot \hat{\boldsymbol{p}}_{2}\right)\right\rangle_{\mathrm{q}}=m_{1} m_{2}\left(\left\langle v_{1 a} v_{2 b}\right\rangle+\left\langle u_{1 a} u_{2 b}\right\rangle\right),
$$

where $a \cdot v_{i}=v_{i a}$, and so on. This gives, after some algebraic manipulation,

$$
\begin{gathered}
\left|\langle\hat{\mathrm{C}}\rangle_{\mathrm{q}}\right| \leq m_{1} m_{2}\left|\left\langle v_{1 a} v_{2 b}\right\rangle+\left\langle v_{1 a} v_{2 b^{\prime}}\right\rangle+\left\langle v_{1 a^{\prime}} v_{2 b}\right\rangle-\left\langle v_{1 a^{\prime}} v_{2 b^{\prime}}\right\rangle\right|+ \\
+m_{1} m_{2}\left|\left\langle u_{1 a} u_{2 b}\right\rangle+\left\langle u_{1 a} u_{2 b^{\prime}}\right\rangle+\left\langle u_{1 a^{\prime}} u_{2 b}\right\rangle-\left\langle u_{1 a^{\prime}} u_{2 b^{\prime}}\right\rangle\right|
\end{gathered}
$$

Now, it is convenient to write

$$
\begin{aligned}
& v_{1 a}=v_{1} \cos \alpha, \quad v_{1 a^{\prime}}=v_{1} \cos \alpha^{\prime}, \\
& v_{2 b}=v_{2} \cos \beta,
\end{aligned}
$$

so that

$$
\begin{gathered}
v_{1 a} v_{2 b}+v_{1 a} v_{2 b^{\prime}}+v_{1 a^{\prime}} v_{2 b}-v_{1 a^{\prime}} v_{2 b^{\prime}}= \\
=v_{1} v_{2}\left[\cos \alpha\left(\cos \beta+\cos \beta^{\prime}\right)+\cos \alpha^{\prime}\left(\cos \beta-\cos \beta^{\prime}\right)\right] .
\end{gathered}
$$

Since the term in square brackets can only take values within the interval $[-2,2]$, we get

$$
-2 v_{1} v_{2} \leq v_{1 a} v_{2 b}+v_{1 a} v_{2 b^{\prime}}+v_{1 a^{\prime}} v_{2 b}-v_{1 a^{\prime}} v_{2 b^{\prime}} \leq 2 v_{1} v_{2} .
$$

Taking the mean value does not alter the inequalities (since $\rho \geq 0$ ), whence

$$
\left|\left\langle v_{1 a} v_{2 b}\right\rangle+\left\langle v_{1 a} v_{2 b^{\prime}}\right\rangle+\left\langle v_{1 a^{\prime}} v_{2 b}\right\rangle-\left\langle v_{1 a^{\prime}} v_{2 b^{\prime}}\right\rangle\right| \leq 2\left|\left\langle v_{1} v_{2}\right\rangle\right|,
$$

which introduced into Equation (21) leads to

$$
\begin{gathered}
\left|\langle\hat{\mathrm{C}}\rangle_{\mathrm{q}}\right| \leq 2 m_{1} m_{2}\left|\left\langle v_{1} v_{2}\right\rangle\right|+ \\
+m_{1} m_{2}\left|\left\langle u_{1 a} u_{2 b}\right\rangle+\left\langle u_{1 a} u_{2 b^{\prime}}\right\rangle+\left\langle u_{1 a^{\prime}} u_{2 b}\right\rangle-\left\langle u_{1 a^{\prime}} u_{2 b^{\prime}}\right\rangle\right| .
\end{gathered}
$$

Finally, a dimensionless inequality is obtained by dividing this last expression by $m_{1} m_{2}\left|\left\langle v_{1} v_{2}\right\rangle\right|$,

$$
|\mathcal{C}| \leq 2+\frac{1}{\left|\left\langle v_{1} v_{2}\right\rangle\right|}\left|\left\langle u_{1 a} u_{2 b}\right\rangle+\left\langle u_{1 a} u_{2 b^{\prime}}\right\rangle+\left\langle u_{1 a^{\prime}} u_{2 b}\right\rangle-\left\langle u_{1 a^{\prime}} u_{2 b^{\prime}}\right\rangle\right|, \quad \text { with } \quad \mathcal{C} \equiv \frac{\langle\hat{C}\rangle_{q}}{m_{1} m_{2}\left\langle v_{1} v_{2}\right\rangle}
$$

It is clear from its derivation that Equation (25) must be satisfied by all quantum bipartite states. Now interestingly, whenever $\rho$ factorizes according to condition 1 , all correlations of the type $\left\langle u_{1 a} u_{2 b}\right\rangle=0$ (for any $\boldsymbol{a}, \boldsymbol{b}$ ) vanish from Equation (25), and we are left with

$$
|\mathcal{C}| \leq 2
$$

Equation (26) is an inequality involving the expectation value of the CHSH-type operator $\hat{\mathrm{C}}$, defined in terms of continuous and commuting variables, and whose violation is a sure manifestation of A-entanglement. The converse is not necessarily true, since it may happen that for a given A-entangled state $\psi$ and for certain specific sets of vectors $\boldsymbol{a}, \boldsymbol{a}^{\prime}, \boldsymbol{b}, \boldsymbol{b}^{\prime}$, Equation (26) holds. However, in line with the 
conclusion arrived at by Gisin [3] (yet in the context of standard Bell inequalities), for any A-entangled state $\psi$ one can always find combinations of $\boldsymbol{a}, \boldsymbol{a}^{\prime}, \boldsymbol{b}, \boldsymbol{b}^{\prime}$ such that Equation (26) is violated.

Equation (25) is conceptually a very interesting result: it tells us that any value of $|\mathcal{C}|$ beyond two is a direct consequence of the quantumness encoded in the diffusive velocity. Recalling that such velocity is intimately related to the dispersive nature of the dynamical variables (see the discussion below Equation (13)), may provide a hint to understand why the violation of Bell inequalities is usually linked to the noncommutativity of the operators involved (see, e.g., refs. [30-33]): noncommuting operators imply a (nonclassical, irreducible) dispersive nature of the corresponding dynamical variables. However, the dispersion of a dynamical variable may be manifested without the need to resort to noncommuting operators, as is the case in Equation (25), in which the dispersive nature is reflected in the presence of the variable $\boldsymbol{u}$.

\section{Concluding Remarks}

Based on the observation that the diffusive velocity is central in describing paradigmatic quantum features (in both the single and the bipartite case), and inspired by the structure of typical Bell inequalities, we have derived a pair of inequalities for a CHSH-type operator involving projections of the momentum operators of the individual particles. The first inequality (Equation (25)) holds for any bipartite system in a pure state, and contains terms related to the diffusive velocities $\boldsymbol{u}_{i}$, confirming that their presence is key in understanding the correlation of bilinear products of the momentum. These additional terms are the ones responsible for violation of the second inequality, Equation (26), which attests the (A-)entanglement of the state. Further, for an A-entangled state each $\boldsymbol{u}_{i}$ depends on the position variables of both particles, and thus the terms responsible for the violation of (26) convey nonlocal information (encoded in the probability distribution of the bipartite state).

These results suggest that by paying due attention to the velocity $\boldsymbol{u}$, it is possible to go deeper into the problem of constructing and understanding Bell inequalities for continuous (momentum) variables. Moreover, the identification of $\boldsymbol{u}$ with the imaginary part of the weak value of the momentum operator, opens the door to explore the role of the weak values of other operators in the establishment of more general Bell inequalities that go beyond the discrete (typically dichotomic) scenario. It must be stressed that the validity of our results is restricted to pure (bipartite) states, and cannot in general be extended to mixed states. In fact, since the very definition of $\left\langle\hat{p}_{i}\right\rangle_{\mathrm{w}}$ involves the pure state $|\psi\rangle$, the velocities $v$ and $\boldsymbol{u}$ (see Equation (5)), and hence all expression involving them, are valid and meaningful only if the system is in a pure state.

Finally, an in-depth analysis of the experimental feasibility of using the momentum variables of entangled particles, as suggested by the above results, would need to be carried out in order to test the degree of violation of the CHSH-type inequality (26). In particular, recent advances in the experimental determination of the real and imaginary part of the weak value of $p$ should be analyzed in detail in light of the present approach.

Author Contributions: All authors contributed equally to this manuscript.

Funding: This research was funded by Dirección General de Asuntos del Personal Académico, Universidad Nacional Autónoma de México, through Project PAPIIT IA101918.

Acknowledgments: The authors wish to express their gratitude to Professors Herman Batelaan and Daniel Cole for their kind invitation to participate in the SED2018 conference in Boston. We gratefully acknowledge financial support from the Fetzer Foundation.

Conflicts of Interest: The authors declare no conflict of interest. The founding sponsors had no role in the design of the study; in the collection, analyses, or interpretation of data; in the writing of the manuscript, and in the decision to publish the results. 


\section{References}

1. Clauser, J.F.; Horne, M.A.; Shimony, A.; Holt, R.A. Proposed Experiment to Test Local Hidden-Variable Theories. Phys. Rev. Lett. 1969, 23, 880-884. [CrossRef]

2. Bell, J.S. Speakable and Unspeakable in Quantum Mechanics; Cambridge University Press: Cambridge, UK, 1988.

3. Gisin, N. Bell's inequality holds for all non-product states. Phys. Lett. A 1991, 154, 201-202. [CrossRef]

4. Valdés-Hernández, A.; de la Peña, L.; Cetto, A.M. Strong entanglement criterion involving momentum weak values. Phys. Lett. A 2018. [CrossRef]

5. Aharonov, Y.; Albert, D.Z.; Vaidman, L. How the result of a measurement of a component of the spin of a spin-1/2 particle can turn out to be 100. Phys. Rev. Lett. 1988, 60, 1351-1354. [CrossRef] [PubMed]

6. Duck, I.M.; Stevenson, P.M.; Sudarshan, E.C.G. The sense in which a "weak measurement" of a spin-1/2 particle's spin component yields a value 100. Phys. Rev. D 1989, 40, 2112-2117. [CrossRef]

7. Nelson, E. Dynamical Theories of Brownian Motion; Princeton U. P.: Princeton, NJ, USA, 1966.

8. de la Peña, L.; Cetto, A.M.; Valdés-Hernández, A. The Emerging Quantum. The Physics Behind Quantum Mechanics; Springer: Berlin, Germany, 2015.

9. Leavens, C.R. Weak measurements from the point of view of bohmian mechanics. Found. Phys. 2005, 35, 469-491. [CrossRef]

10. Wiseman, H.M. Grounding bohmian mechanics in weak values and bayesianism. New J. Phys. 2007, 9, 165-177. [CrossRef]

11. Kocsis, S.; Braverman, B.; Ravets, S.; Stevens, M.J.; Mirin, R.P.; Shalm, L.K.; Steinberg, A.M. Observing the average trajectories of single photons in a two-slit interferometer. Science 2011, 332, 1170-1173. [CrossRef] [PubMed]

12. Braverman, B.; Simon, C. Proposal to Observe the Nonlocality of Bohmian Trajectories with Entangled Photons. Phys. Rev. Lett. 2013, 110, 060406. [CrossRef] [PubMed]

13. Traversa, F.L.; Albareda, G.; Di Ventra, M.; Oriols, X. Robust weak-measurement protocol for Bohmian velocities. Phys. Rev. A 2013, 87, 052124. [CrossRef]

14. Mahler, D.H.; Rozema, L.; Fisher, K.; Vermeyden, L.; Resch, K.J.; Wiseman, H.M.; Steinberg, A. Experimental nonlocal and surreal Bohmian trajectories. Sci. Adv. 2016, 2, e1501466.

15. Hiley, B.J. Weak values: Approach through the Clifford and Moyal algebras. J. Phys. Conf. Ser. 2012, 361, 012014. [CrossRef]

16. Flack, R.; Hiley, B.J. Feynman Paths and Weak Values. Entropy 2018, 20, 367. [CrossRef]

17. Dressel, J.; Malik, M.; Miatto, F.M.; Jordan, A.N.; Boyd, R.W. Colloquium: Understanding quantum weak values: Basics and applications. Rev. Mod. Phys. 2014, 86, 307. [CrossRef]

18. Dressel, J.; Jordan, A.N. Significance of the imaginary part of the weak value. Phys. Rev. A 2012, 85, 012107. [CrossRef]

19. Cohen. E.; Pollak, E. Determination of weak values of quantum operators using only strong measurements. Phys. Rev. A 2018, 98, 042112. [CrossRef]

20. Dressel, J.; Jordan, A.N. Contextual-value approach to the generalized measurement of observables. Phys. Rev. A 2012, 85, 022123. [CrossRef]

21. Bohm, D. A Suggested Interpretation of the Quantum Theory in Terms of “Hidden" Variables I. Phys. Rev. 1952, 85, 166-179. [CrossRef]

22. Bohm, D. A Suggested Interpretation of the Quantum Theory in Terms of "Hidden" Variables II. Phys. Rev. 1952, 85, 180-193. [CrossRef]

23. Holland, P.R. The Quantum Theory of Motion; Cambridge University Press: Cambridge, UK, 1993.

24. Nelson, E. Quantum Fluctuations; Princeton U. P.: Princeton, NJ, USA, 1985.

25. de la Peña, L. New Formulation of Stochastic Theory and Quantum Mechanics. J. Math. Phys. 1969, 10, 1620. [CrossRef]

26. de la Peña, L.; Cetto, A.M. Does quantum mechanics accept a stochastic support? Found. Phys. 1982, 12, 1017-1037. [CrossRef]

27. Bohm, D.; Hiley, B.J. Non-locality and locality in the stochastic interpretation of quantum mechanics. Phys. Rep. 1989, 172, 93-122. [CrossRef]

28. Takabayashi, T. On the Formulation of Quantum Mechanics associated with Classical Pictures. Prog. Theory Phys. 1952, 8, 143-182. [CrossRef] 
29. Hirschfelder, J.O.; Christoph, A.C.; Palke, W.E. Quantum mechanical streamlines. I. Square potential barrier. J. Chem. Phys. 1974, 61, 5435-5455. [CrossRef]

30. Cirel'son, B.S. Quantum generalizations of Bell's inequality. Lett. Math. Phys. 1980, 4, 93-100. [CrossRef]

31. Revzen, M.; Lokajícek, M.; Mann, A. Bell's inequality and operators noncommutativity. Quantum Semiclass. Opt. 1997, 9, 501. [CrossRef]

32. Landau, L.J. On the violation of Bell's inequality in quantum theory. Phys. Lett. A 1987, 120, 54-56. [CrossRef]

33. Fritz, T. Nonlocality with less complementarity. Phys. Rev. A 2012, 85, 022102. [CrossRef]

(C) 2019 by the authors. Licensee MDPI, Basel, Switzerland. This article is an open access article distributed under the terms and conditions of the Creative Commons Attribution (CC BY) license (http:// creativecommons.org/licenses/by/4.0/). 